nördliche Teil von Groß-Obi und einige andere Inseln der Obi-Gruppe durch die große Verbreitung von verschiedenen Intrusiv- und EffusivGesteinen (u. a. viel Serpentinen) mehr Übereinstimmung mit den nördlichen Molukken (Halmahera usw.) zeigen und somit nicht zu der Reihe: Sula-Obi-Misol gehören. Wichtig ist ferner, daß die charakteristische geodenführende Schieferfazies des mittleren Dogger von Obi auf Misol weder in diesem noch in einem anderen jurassischem Horizonte bekannt ist. Misol kann infolgedessen mit der Obi-Gruppe nicht zu einer geologischen Einheit zusammengefaßt werden; Misol und Obi können nicht Teile ein und derselben Faltungskulisse sein, wịe vaN $\mathrm{Es}^{1}$ ) neuerdings annimmt.

\title{
Die Beziehungen zwischen Schwereanomalien und Bau der Erdrinde.
}

\author{
Von Franz Kossmat (Leipzig).
}

(Mit 2 Textfiguren.)

\section{Allgemeine Vorbemerkungen ${ }^{2}$ ).}

Die Schwerestörungen des Festlandes drücken sich in der Abweichung der aus Pendelbeobachtungen ermittelten örtlichen Werte der Beschleunigung durch die Schwere gegenüber einem theoretischen Normalwerte aus. Zu ihrer Darstellung werden an der beobachteten Beschleunigung (ausgedrückt in Metern oder in Zentimetern pro Sekunde) Reduktionen nach verschiedenen Gesichtspunkten und Methoden angebracht. Bei der Verwertung der Ergebnisse ist daher immer eine Angabe darüber nötig, welche Art von Schwereanomalie gemeint ist.

1. Der Reduktionswert $g_{0}$ ergibt sich aus der Umrechnung des Beobachtungswertes $g$ auf das Meeresniveau nach der Näherungsformel

$$
g_{0}=g+\frac{2 H}{R} g=g+0.0003086 H
$$

( $g$ in Zentimetern, Seehöhe $H$ und mittlerer Erdradius $R$ in Metern).

Es liegt dieser vereinfachten Methode die Annahme zugrunde, da $\beta$ die Höhe $H$ zum Meeresspiegel herabgerückt ist und der "in freier Luft « darüber befindliche Beobachtungspunkt zum gleichen Niveau herab-

1) vav Es, L. J. C., De Tektoniek als Basis voor de opsporing von Ertsen en fossile Brandstoffen. Alg. Ingen. Cong. Batavia. 1920.

2) Die Abschnitte I, II, IV stützen sich auf die ansführlichere Arbeit des Autors: Die mediterranen Kettengebirge in ihrer Beziehung zum Gleichgewishtszustand der Erdrinde. Abh. Sächs. Akad. d. Wiss. Bd. XXXVIII, 2. Leipzig 1920. 62 S. 1 Karte. Manche Ergänzungen allgemeiner Art sind durch den seither erfolgten Gedankenaustausch mit Fachmännern der geophysikalischen Richtung angeregt. 
gezogen wird. Bei unregelmäßiger Geländegestaltung ist zu berücksichtigen, daß die beobachtete Schwere etwas kleiner ist als auf gleich hoher Ebene, da Berge über dem Beobachtungsort und Taleinschnitte unter ihm der Beschleunigung entgegen wirken. Man muß daher auf eingeebnetes Gelände umrechnen und erhält die immer positive "orographische Korrektion $« g^{\prime}-g$, deren Hinzufügung $z u g_{0}$ den Wert $g_{0}^{\prime}$ liefert.

2. $g_{0}^{\prime \prime}$ ist der sogenannte Bouguersche Reduktionswert, den man erhält, wenn von $g_{0}$ bzw. $g_{0}^{\prime}$ die Attraktionswirkung der zwischen Meeresniveau und Beobachtungsort liegenden Gesteinsplatte abgezogen wird. Das Erdrelief ist hier durch Fortschaffung der Masse beseitigt gedacht.

$$
g_{0}^{\prime \prime} \text { ist somit }=g+\frac{2 H}{R} g+\left(g^{\prime}-g\right)-\frac{3}{2} \cdot \frac{D}{D m} \cdot \frac{H}{R} g
$$

( $D=$ spez. Gewicht der Gesteinsmasse unter dem Beobachtungspunkt, $D m=$ spez. Gewicht der Erde = 5.52.)

3. $\gamma_{0}$ ist die für Meeresniveau geltende Normalschwere, die dem Beobachtungsort gemäß seiner geographischen Breitenlage bei gleichmäßiger Massenschichtung des Erdkörpers und Fehlen jedes Reliefs zukäme. Streng genommen bezieht sich $\gamma_{0}$ auf ein Rotationsellipsoid (das Referenzellipsoid), während die bisher erwähnten Reduktionswerte $g_{0}, g_{0}^{\prime}, g_{0}^{\prime \prime}$ für die Geoidoberfläche gelten, die sich infolge der nicht völlig gleichmäßigen Massenschichtung vom Ellipsoid etwas nach oben und unten entfernt. In den Tiroler Alpen beträgt nach HELMERT, Zeitschr. d. Ges. f. Erdkunde, Berlin 1913, S.28, die Geoiderhebung etwa 13 m, in Zentralasien ein Mehrfaches davon; die Abweichung bleibt aber so klein, daß sie, besonders in Europa, für unsere Zwecke nicht ins Gewicht fällt.

Die Normalformel wurde von HELMERT durch Mittelbildung aus einer großen, auf Wien als Ausgangsstation bezogenen Beobachtungsreihe ermittelt und lautete in der Fassung von 1901:

$$
\gamma_{0}=978,046\left(1+0.005302 \sin ^{2} \varphi-0.000007 \sin ^{2} 2 \varphi\right) \text {, }
$$

wo $\varphi$ die geographische Breite, 978,046 die Normalschwere am Äquator, ausgedrückt in Zentimetern Beschleunigung pro Sekunde, bedeutet.

Später kam die auf Potsdam als Ausgangsstation bezogene Formel

$$
\gamma_{0}=978,030\left(1+0.005302 \sin ^{2} \varphi-0.000007 \sin ^{2} 2 \varphi\right)
$$

zustande, die den Tabellen von Borrass 1909 und 1912, sowie den neueren Rechnungen von HaASEManN für Deutschland (1916) zugrunde gelegt ist. Die dort gegebenen Zahlen wurden daher auch auf der zu meiner Arbeit beigegebenen Karte verwendet.

Vergleicht man die Reduktionswerte der beobachteten Schwerebeschleunigungen mit dem »Normalwert «, so erhält man die Schwereanomalien ${ }^{1}$ ).

1) Selbstverständlich werden bei einer Vergrößerung der Ziffer für die Normalschwere, wie sie wiederholt wahrscheinlich gemacht wurde, die Anomalien eino Verschiebung nach der negativen Seite erfahren. KoHLSCHÜTrTER neigt auf Grund 
a) $g_{0}-\gamma_{0}=\Delta g$ bedeutet die totale Schwerestörung. Dieser Wert bildet den Ausgang für die Isostasiefrage, gibt aber nicht ein ohne weiteres brauchbares Bild. Er ist unter gleichen Bedingungen auf dem Berge größer als im Tale; es ist daher in stark zerschnittenem Gelände notwendig, die mittlere Gebirgshöhe zugrunde zu legen. Außerdem unterliegt aber die Schwere eines Gebietes auch der Beeinflussung durch Massenstörungen, die mit dem übrigen Erdrelief zusammenhängen.

b) Aus dem genannten Grunde führten HAYFORD und BowIE für die Vereinigten Staaten an Stelle der $g_{0}-\gamma_{0}$ die verbesserten Schwere anomalien ( $g$ beobachtet $-g$ berechnet) ein, wobei sie in der Berechnung die störenden Einflüsse des Erdreliefs unter Zugrundelegung der Isostasietheorie zu ermitteln suchten (S. 182 dieser Arbeit).

c) $g_{0}^{\prime \prime}-\gamma_{0}=A g^{\prime \prime}$ kann als Bouguersche Anomalie bezeichnet werden und gibt einen Hinweis auf die Dichtestörungen unter der Geoidoberfläche, da die über letztere aufragenden Massen beseitigt gedacht sind. Für sich allein geben diese Anomalienziffern freilich keinen Hinweis auf die Lage der unterirdischen Massenstörungen innerhalb des Erdkörpers. Die Einschränkung der in Betracht kommenden Möglichkeiten ergibt sich aber sofort, wenn man den Zusammenhang mit den Strukturelementen beobachtet. In dieser Beziehung sind gerade die Verhältnisse in Mitteleuropa ein glänzender Beleg dafür, daB der Hauptsitz der Anomalien noch innerhalb der tek. tonisch bewegten Erdrinde zu suchen ist.

Positives $\Delta g^{\prime \prime}$ weist auf zu große Dichte (Massenüberschuß), negatives auf zu kleine Dichte (Massendefizit) unter dem Meeresniveau hin. Das Beobachtungnetz von Mitteleuropa, einschließlich Dänemark und Italien, ist fein genug, um die Darstellung dieser für die Tektonik bedeutungsvollen Verhältnisse in Form einer Isanomalenkarte zu gestatten, von der auf S. 175 ein verkleinerter, die Hauptkurven wiedergebendex Auszug abgedruckt ist.

Die Defizite und Úberschüsse werden oft durch Angabe der »ideellen störenden Masse « ausgedrückt. Eine Beschleunigungsdifferenz von $0,001 \mathrm{~cm}(=$ eine Einheit der 5. Dezimale der in Metern dargestellten Beschleunigung) entspricht der Anziehung einer auf Meeresniveau kondensiert gedachten, ebenen, unendlich ausgedehnten Gesteinsplatte von $10 \mathrm{~m}$ Mächtigkeit und etwa 2,4 Dichte. Da die wirklich fehlende oder

der Mittelwerte seiner afrikanischen Messungen zur Annahme, daß eine Erhöhung der Äquatorkonstante um etwa fünf Einheiten der letzten Dezimale, also auf 978,035 $\mathrm{cm}$ vorzunehmen sei, HAYFORD und BowTE halten die Ziffer 978,038 für wahrscheinlich. HELMERT und BERROTH kamen in neueren Berechnungen sogar zu einem noch etwas höheren Wert, der wieder ungefähr dem der Wiener Formel entsprechen würde (HeLMerT, Sitzungsber. d. preuß. Akad. 1915. II, S. 676). Da die örtlichen Anomalien dadurch in gleicher Weise betroffen werden und somit die Isanomalenkurven keine Formveränderung erfahren, ist es wohl geraten, einstweilen bei den Borrassschen Tabellenziffern zu bleiben, um keinen Anlaß zu Verwechslungen zu geben. 
in Uberschuß vorhandene Gesteinmasse sich auf eine gewisse Tiefe der Erdrinde, die mindestens nach Zehnern von Kilometern zählt, verteilt, muß sie zur Erzielung des beobachteten Effekts größer sein als die auf Meeresniveau kondensierte und so dem Beobachtungspunkt nahe gerückte ideelle Masse. (HELmERT, Enzyklopädie der mathematischen Wissenschaften, Bd. VI, 1. B., Heft 2, S. 106). Für die Veranschaulichung der zur Kompensation nötigen Massen ist aber diese Art der Darstellung brauchbar.

Naturgemäß kommen in den Werten $g_{0}^{\prime \prime}-\gamma_{0}$ und $g_{0}-\gamma_{0}$ nicht nur die senkrecht unter dem Beobachtungsorte liegenden Massenver-

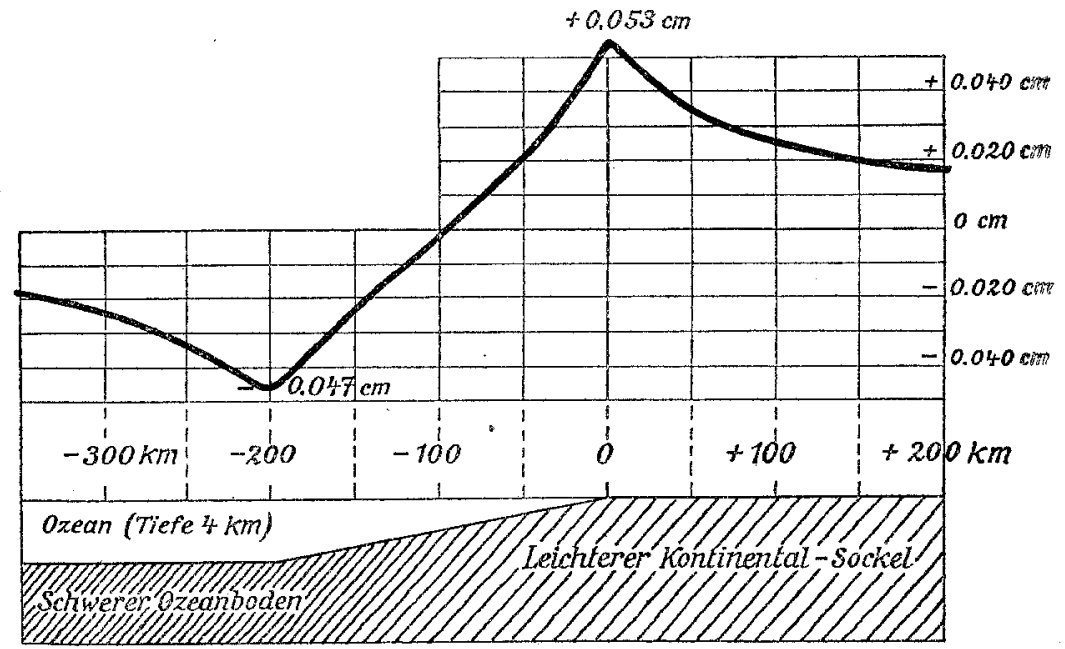

Fig. 1. Kurve der Kontinentalrandstörung unter Voraussetzung det Isostasie (nach Hexmert 1909). Angenommene Ausgleichstiefe $118 \mathrm{~km}$.

Die durch Aneinandergrenzen des tiefliegenden schweren Ozeanbodens und der ansteigenden leichteren Kontinentalplattform verursachte Aländerung des örtlichen Schwerewertes erreicht ibr positives Maximum am Rande der Kontinentalplattform, ihr negatives am Fuß des Steilabfalls zur Tiefsee. Die Schwerestörungen sind in tausendstel Zentimeter Beschleunigung pro Sekunde dargestellt. Darunter: das schematische Krustenprofil.

hältnisse zum Ausdruck, sondern es muß dort, wo Krustenteile verschiedener Dichte aneinander grenzen, der beiderseitige Einfluß auf eine gewisse Entfernung bemerkbar sein ${ }^{1}$ ), die mit der Tiefenlage der Massenstörung zunimmt. Wichtig ist dies bei den Schwerestörungen des Kontinentalrandes, die vom dichteren Ozeanboden her erheblich im positiven

1) Auf der Isanomalenkarte erscheinen z. B. jene Massenüberschüsse, di $\theta$ offenkundig dem Sehwarzwald und den Vogesen entsprechen, in eigenartiger Weise nach NW verschoben, während jene des Harzes und des Magdeburger Horsts an der richtigen Stelle sitzen. Vielleicht führen sich die ersterwähnten Verschrebungen auf die Fernwirkung des großen unterirdischen Alpendefizits zurück. 
Sinne beeinflußt sind, während andererseits auf dem angrenzenden Meeresstreifen eine Schwereminderung durch die Einwirkung des leichteren Kontinentalsockels eintreten maß (siehe HrLMERTs theoretische Kurve, Fig. 1). In Mitteleuropa ist diese Einwirkung glücklicherweise höchstens einige Einheiten; aber auch im Mittelmeer tritt der Einfluß gegenüber den örtlichen Störungen zweifellos stark zurück, sonst könnten sich nicht Küsten, die einander unmittelbar gegenüber liegen, ganz verschieden verhalten; man vergleiche auf der Karte Korsika mit der Riviera, oder die italienische Ostküste mit Dalmatien. Jedenfalls wäre es ein gewagtes Unternehmen, aus den $g_{0}^{\prime \prime}-\gamma_{0}$ diese theoretischen Einflüsse ausmerzen zu wollen und daraufhin eine Karte zu entwerfen. Noch mehr gilt dies von der Berücksichtigung anderer isostatischer Reduktionen auf Grund des Festlandreliefs. worauf besonders KOHLSCHÜTTER in seiner Arbeit über Deutsch-Ostafrika hinwies. Es empfiehlt sich, die Bouguerschen Anomalien darzustellen, wie sie sich zeigen, und die verschiedenen Möglichkeiten bei der Interpretation zu berücksichtigen.

Das nach einheitlichem Prinzip durchgerechnete Zahlenmaterial für die ganze Erde gibt E. Borrass: Bericht über die relativen Messungen der Schwerkraft mit Pendelapparaten in der Zeit von 1808-1909 und ihre Darstellung im Potsdamer Schweresystem. Verb. der vom 21. bis 29. Sept. 1909 in London und Cambridge abgehaltenen XVI. allgem. Konf. f. internat. Erdmessung. Berlin 1912. III. Teil. 288 Seiten mit Karten.

E. Borrass: Bericht über die relativen Messungen der Schwerkraft mit Pendelapparaten für den Zeitraum von 1909-1912. Verh. d. vom 17.-27. Sept. 1912 in Hamburg abgehaltenen XVII. allg. Konf. f. internat. Erdmessung. Berlin 1914. II. Teil B. XI. S. 261-343.

Um eine Übersicht des Verhaltens der verschiedenen Reduktionswerte zu geben, seien hier aus diesen Tabellen die Ziffern für das Geodätische Institut Potsdam und für den Säntis mitgeteilt ( $g$ dargestellt in Zentimetern pro Sekunde; $\Delta g, \Delta g^{\prime}, \Delta g^{\prime \prime}$ in $0.001 \mathrm{~cm}$ ).

\begin{tabular}{|c|c|c|c|c|c|}
\hline & Seehöhe & $\begin{array}{l}\text { Gesteins- } \\
\text { dichte }\end{array}$ & $g$ & $g_{0}$ & $g^{\prime}-g$ \\
\hline $\begin{array}{l}\text { Potsd a m, geodät. Institut } \\
52^{\circ} 22.9^{\prime} \text { n. B., } 13^{n} 4.1^{\prime} \text { ö. L.) } \\
\text { Sän ti s, Schweiz } \\
\left(47^{\circ} 15.1^{\prime} \text { n. B., } 9^{\circ} 20.6^{\prime} \text { ö. L.) }\right.\end{array}$ & $\begin{array}{r}87 \mathrm{~m} \\
2500 \mathrm{~m}\end{array}$ & 2.0 & $\begin{array}{r}981,274 \\
980,138\end{array}$ & $\begin{array}{l}981,301 \\
980,910\end{array}$ & $\begin{array}{r}0 \\
+64\end{array}$ \\
\hline
\end{tabular}

\begin{tabular}{l|c|c|c|c}
\hline & $g_{0}^{\prime \prime}$ & $\gamma_{0}$ & $\Delta g^{\prime \prime}=g_{0}^{\prime \prime}-\gamma_{0}$ & $\Delta g=g_{0}-\gamma_{0}$ \\
\hline $\begin{array}{l}\text { Potsdam, geodät. Institut } \\
\left(52^{\circ} 229^{\prime} \text { n. B., 130 4.1' ö. L. }\right)\end{array}$ & 981,294 & 981,277 & +17 & +24 \\
$\begin{array}{l}\text { Sänt is, Schweiz } \\
\left(47^{\circ} 15.1^{\prime} \text { n. B., 90 } 20.6^{\prime} \text { ö. L. }\right)\end{array}$ & 980,691 & 980,820 & -129 & +90
\end{tabular}




\section{Europäische Beobachtungen.}

\section{A. Die Schwereanomalien in den mediterranen Kettengebirgen.}

1. Die negativen $A g^{\prime \prime}$ (Massendefizite) unter den jungen Kettengebirgen sind nicht die Folge einer Lockerung des Materials, sondern sie werden verursacht durch die Bildung eines Wulstes von leichten Gesteinen infolge des Zusammenschubs der Oberkruste und der gleichzeitig in der Tiefe vor sich gehenden Ansammlung leichter "salischer " Magmamassen. Der so gebildete Wulst sinkt in die plastische schwerere Unterlage ein und drängt einen entsprechenden Teil der letzteren beiseite, so daß unter dem Gebirge eine Dichtesynkline entsteht. Ein Hinweis auf die Richtigkeit dieser sehon von OSMOND FIsHer 1881 vertretenen Auffassung ist $u$. a. dadurch gegeben, daß die Defizitzonen der Kettengebirge begleitet werden von deutlich damit in Kausalzusam menhang stehenden Utberschußgürteln.

a) In den Westalpen ${ }^{1}$ ) folgt die Zone der größten Defizite (Dichtesynkline) dem tiefeingefalteten Briançonnaisgürtel. Am Südgehänge des oberen Rhonetals wird der Wert von - 145 Einheiten erreicht, bei Chamonix -123, unter dem Mont Blanc -218. Dagegen bezeichnet die weiter südlich liegende Biella-Ivreagegend eine zwischen dem alpinen und dem apenninischen Defizit aufsteigende Dichteantiklinale (bis +80 Einheiten der letzten Dezimale von $g$ ). An sie schließt sich weiter nach Osten die Schwelle von Verona und das große adriatische Oberschußgebiet an, das am Monte Gargano +132 Einh. aufweist.

b) In den Ostalpen liegt das größte Massendefizit unter der Zentralzone und schwächt sich mit der Verbreiterung des Gebirges gegen Osten ab, so daß am Rande der pannonischen Senke stellenweise sogar ein Uberschuß von Osten her eindringt. Diese Erscheinung läßt sich nicht vereinen mit der Auffassung, daß die ostalpine Zentralzone ein über den hohen Tauern liegendes System von Schubdecken bildet, das den angrenzenden Teil der pannonischen Ebene unterlagert. Es müßte in diesem Falle die Verstärkung der leichten Oberkruste durch diese Deckenanhäufung von einem Wachsen der Defizite östlich des Tauernfensters begleitet sein, während das Gegenteil eintritt. Die Frage der tektonischen Stellung der ostalpinen Zentralzone bedarf auch aus diesem Grunde einer eingehenden Neuüberprüfung, und es werden in der ausführlicheren Arbeit, Abh. d. sächs. Akad. d. Wiss., XXXVIII, 2., S.13-17, die Gründe auseinander gesetzt, die dafür sprechen, daß der östliche Teil der Zentralalpen autochthon ist, und $\mathrm{daB}$ am Nordrande der Hohen Tauern südgerichtete Uberschiebungen (besser gesagt: Unterschiebungen

1) Eine geologische Deutung der zahlreichen Schweremessungen in den Schweizer Alpen gibt AxB. Horm an mehreren Stellen, zuletzt in der Geologie der Schweiz, II. Bd., I. Heft. Leipzig 1919. Er bringt sie in Zusammenhang mit dem Decken. bau. Auf die Magmabewegungen unter dem Kettengebirge geht er nicht sein. 
von Seite der südlicher liegenden Zonen) vorliegen, die jenen am Südrande der italienischen Kalkalpen in der Entstehungsart und auch im Ausstrichbilde verwandt sind.

c) In den Faltengebirgen, die innere Senkungsfelder, so das westliche Mittelmeer und die pannonische Ebene umgürten, also im Apennin, im Atlas, in den Andalusischen Ketten und in den Karpathen rückt das größte Massendefizit nahe an den Außenrand des Gebirges, während in dessen inneren Teilen, ebenso wie im Senkungsfeld, Massenüberschuß herrscht. Diese Tatsache ging schon aus den bahnbrechenden Beobachtungen v. STERNECKs (1893/94) in Osterreich-Ungarn hervor.

2. Gegen die nördlich von ihnen liegenden Rumpf- und Tafelländer sind die mediterranen Kettengebirge Europas durch eine von tertiären und quartären Ablagerungen erfüllte Randsenke, das sogenannte Vorland begrenzt. Die Randsenken sind ein bei der Faltung durch die Last des entstebenden Gesteinswulstes mit hinabgezogener Teil der starren Einfassung der Kettenregion und zeigen daher Massendefizit $\left(-\Delta g^{\prime \prime}\right)$, das nicht selten, so in Galizien und im Atlasvorlande, auch eine absolute Schwerestörung negativer Art $(-\Delta g)$ bedingt. Häufig lösen sie sich infolge der vom einsinkenden Wulst ausgehenden Spannung durch einen Bruch, z. B. den Rhone-, Donau-, Quadalquivir-Bruch, vom weiter entfernten Teile des Außengebietes ab.

Das Kettengebirge wird somit nicht für sich allein, sondern in Verbindung mit seinem Vorland kompensiert. Die Rechnung ergibt z. B. für die Alpen trotz der großen, unter der Geoidfläche liegenden Massendefizite einen absoluten Schwereüberschuß, da die über das Meeresniveau aufragende Gebirgsmasse größer ist als das Defizit unter ihr. Bei einem rohen Uberschlag dieser Art kommen rund +70 Einheiten der letzten Dezimale von $g$ heraus, was zunächst überrascht. Doch ist zu erwägen: 1. Unter den Alpen liegen nicht ausschließlich große Defizite, sondern auch einige Überschußgebiete. 2. Der absolute Schwereüberschuß ist nicht mit der Isostasiestörung identisch, -da Masseneinwirkung von außen her etwas beteiligt ist (N. B. dies hätte auch in meiner Arbeit in Abh. sächs. Akad. 1920, S. 7 und 20 angeführt werden sollen). Allerdings ist die hier hauptsächlich in Betracht kommende "Kontinentalrandstörung " für Mitteleuropa höchstens einige Einheiten, und sie scheint für die Alpen auch in Mittelmeernähe nicht bedeutend zu sein, da sich die Isanomalen in ihrem Verlauf und in ihren Werten von den tektonischen Hauptzonen und nicht von der Meeresnähe abhängig zeigen. 3. $\gamma_{0}$ kann zu klein sein, wie in den Vorbemerkungen gesagt wurde.

Immerhin zeigen auch die mit allen Vorsichtsmaßregeln ausgeführten Berechnungen HAYFords und BowIEs für die Rocky Mountains, daß ein Uberschuß von einigen Zehnern der Anomalieeinheit auch dort bleibt.

Die letzten Hebungen im Alpenvorland, die zur Trockenlegung und teilweisen Zerschneidung fübrten, sind daher nicht einfach als isosta- 
tische Ausgleicherscheinungen infolge der Gebirgsabtragung aufzufassen - wenn sie auch dadurch begünstigt wurden -, sondern hängen mit der ausklingenden Alpenfaltung zusammen, die einer vollen isostatischen Anpassung entgegenwirkt. Vgl. z. B. die noch in der Diluvialzeit erfolgten Schrägstellungen von Terrassen und Talböden im Piave-, Tagliamento- und Isonzogebiet (PENCK und BrüCKNER: Die Alpen im Eiszeitalter, III. Bd.; Kossmat: Morpholog. Entwickl. des Isonzo- und oberen Savegebiets, Zeitschr. d. Ges. f. Erdk., Berlin 1916. S. 55).

3. Die Adria ist keine Randsenke, sondern ein zwischen den apenninischen und dinarischen Ketten eingeengter Rest der südmediterranen Geosynklinale. Sie steht unter dem Einfluß tiefliegender Massenzuwanderung aus den beiderseitigen Gebirgsgürteln und ist daher ein noch in teilweiser Senkung begriffenes UberschuBgebiet.

4. Die an Verbreiterungen des mediterranen Kettengürtels gebundenen und rings von Faltenzügen umschlossenen Innensenken vom Typus des tyrrhenischen und pannonischen Beckens sind MassenüberschuBgebiete und zeigen starkes Niedergehen am Ende des Faltungszyklus. In ihren Untergrund scheint ein großer Teil der bei Ausbildung der Dichtesynkline im umrahmenden Hauptfaltengürtel verdrängten schweren Tiefenmagmen abgewandert zu sein. Letztere müssen sich hier Platz geschafft haben auf Kosten salischer Massen, die dabei, vermutlich über sie hinweg, aus der Innenregion gegen die Umwallung ausgebreitet werden.

Durch ihren Massen- und Schwereüberschuß, ihre vulkanischen Phänomene und ihre unregelmäßig in die Faltengürtel eingreifenden Umrisse unterscheiden sich die Innenbecken von den Randbecken des alpin-karpathischen Vorlandtypus.

Beim Nachsinken der überlasteten tyrrhenischen und pannonischen Region muß in der Tiefe eine gegen die Außenketten gerichtete Rückverschiebung dichter Massen eintreten, so daß der Utberschuß teilweise die angrenzenden Partien der Faltenumrandung erobert und sie mit in den Einbruch zieht. So gibt uns die heutige Dichteverteilung unter den karpathischen und apenninischen Gebirgen ein Bild, das bereits stark von dem ursprünglichen abweicht, weil der Ausgleichsprozeb in vollem Gang ist.

Die junge Senkung der dalmatinischen Küste scheint mit der vom tyrrhenischen Einbruch ausgehenden Wanderung einer großen wellenförmigen Biegung der Gleichgewichtsfläche zusammenzuhängen. Nach dieser Auffassung ist der Schwereüberschuß hier nachträglich von Westen her in die äußeren dinarischen Falten eingedrungen.

Die Innensenken können im Laufe des Ausgleichsvorganges mit dem Vorland in Verbindung treten, so daß stellenweise der Zusammenhang der Kettengebirge im orographischen Bilde nachträglich unterbrochen wird. Selbstverständlich wird es auch Gebiete geben, die schon während der Faltung nicht geschlossen über den Meeresspiegel stiegen. 
Wie bei obigen Ausführungen bereits erwähnt wurde, hat die in Verbindung mit dem Faltungsvorgange erfolgte Störung der Massenschichtung innerhalb der tiefgelegenen Rindenzonen eine Wellenform, da die Defizite unter dem Gebirge und Vorland notwendigerweise von entsprechend ausgedehnten Uberschußgürteln ${ }^{1}$ ) begleitet werden. Der in letzteren einsetzende Senkungsvorgang überdauert die Faltung und währt so lange, bis der Dichteüberschuß durch Anhäufung spezifisch leichter Sedimente im sinkenden Raume aufgezehrt ist. Derartige Senken gehören zu den Geosynklinalen erster Ordnung, die nach entsprechend langer Zeit bekanntlich einen neuen Faltungszyklus auslösen können. Es ist also nicht nur, wie man im allgemeinen seit Dana annimmt, die geosynklinale Sedimentbildung eine Vorbedingung der Faltung, sondern umgekehrt zieht auch jede Faltung durch die Veränderung des Gleichgewichtszustandes, die sie mit sich bringt, neue Geosynklinalbildungen nach sich. Wir können dieses Wechselspiel zurückverfolgen bis in vorpaläozoische Zeit und gewinnen den Findruck, daß die Beschränkung der Hauptgeosynklinalen und Hauptfaltenketten auf einige wenige Gürtel und ihr langrhythmischer Ablauf Ausleseerscheinungen sind, die sich im Laufe langer Zeiträume mehr und mehr herausgebildet haben und mit einer zunehmenden Versteifung der Erdrinde in $\mathrm{Zu}$ sammenhang stehen.

Die durch Massendefizit ausgezeichneten Randsenken der mediterranen Kettengebirge sind Geosynklinalen zweiter Ordnung, deren Vertiefung an den Faltungsvorgang unmittelbar gebunden ist und mit ihm erlischt. Darin liegt ein erheblicher Unterschied gegenüber den Geosyklinalen erster Ordnung, an die sich jene Erscheinung knüpft, die wir als "Wandern der Faltengïrtel « bezeichnen. (Vgl. den teilweisen Wechsel des Schauplatzes der kaledonischen, variskischen und alpinmediterranen Faltung Europas.)

\section{B. Die Schwereanomalien des außeralpinen Mitteleuropa.}

Die karbonischen Faltengebirge Mitteleuropas waren tektonisch den jungen Ketten des Mittelmeergebiets und anderer Teile der Erde völlig analog. Sie besaßen wie sie sowohl Rand- wie Innensenken mit entsprechender geologischer Charakteristik und müssen daher seinerzeit Dichteanomalien aufgewiesen haben, die den gleichen Gesetzen folgten wie in den jungen Kettengebirgen. Diese Art von Störungen ist aber heute in ihnen teils verlöscht, teils völlig umgeprägt ${ }^{2}$ ). Die Isanomalen-

1) Es ist anzunehmen, daß letztere jeweils während der Hochstadien der Faltung besonders stark betont sind, dann aber allmählich breit laufen, wie dies etwa ein rasch erzeugter Wulst in einer teigigen Substanz machen würde. Es scheint nicht aussichtslos, stratigraphicehe Oszillationen auf diese Fragen hin zu untersuchen.

2) Einen solchen umgeprägten Defizit- Rumpf « aus dem variskischen Gebirge glaube ich in der böhmischen Masse erkennen zu können. Vgl. die Isanomalenkarte. 
karte der $\Delta g^{\prime \prime}$ des außeralpinen Mitteleuropa erklärt sich durch die postvariskischen Vorgänge, denen die Horste und Gräben Süd- und Mitteldeutschlands, sowie die saxonischen Falten Nordwestdeutschlands ihre Entstehung verdanken. Die Anomalien sind mithin zeitlich in hohem Grade veränderlich.

Da die alte Faltentektonik der variskischen Rumpfgebirge in der unseren Beobachtungen zugänglichen Oberkruste unverändert erhalten ist, muß man auch aus dieser Erscheinung zu dem Schluß kommen, daß der Sitz der Schwerestörungen unter der letzteren gelegen ist. Er ist aber andererseits nicht in unbegrenzter Tiefe zu suchen, sonst könnten nicht so kleine Züge des Krustenbaues, wie die deutschen Horste und Gräben sind, in den $\Delta g^{\prime \prime}$ so klar zum Ausdruck kommen. Dies, zusammengenommen mit der Tatsache des stattgefundenen Ausgleichs der alten Dichtewellen, weist deutlich auf die hohe Plastizität der tieferen Rindenpartie hin, beliräftigt also unsere Vorstellungen von der Existenz einer Magmaschale.

In der Isanomalenkarte Deutschlands verhalten sich tektonische Depressionen, wie Rheingraben, Leinegraben, fränkische Mulde und norddeutsche Senke als "Dichtesynklinen " und stellen im allgemeinen Defizitgebiete dar. Die Horste vom Schwarzwald-, Vogesen-, Harzund Bornholmtypus sind hingegen ausgesprochene Dichteschwellen, also Aufwölbungen der schwereren Untergrundmassen. Sie unterscheiden sich dadurch grundsätzlich von Faltenketten alpiner Art. Ihre Kompensation erfolgt ausschließlich durch Verbindung mit angrenzenden Defizitgebieten und deutet weitgehende, elastische Utbertragung in einer verhältnismäßig steifen Kruste an. Die ziemlich großen Dichteanomalien des außeralpinen Mitteleuropa sind von systematischer Bedeutung auch deshalb, weil sie sich unter einem schwachen Erdrelief einstellen, das in Norddeutschland und Dänernark überhaupt nicht in Betracht kommt. Hierdurch vereinfacht sich die Frage nach der Ursache ganz ungemein. Der Einfluß des Ozeanbodens beträgt für Mitteleuropa äußerstenfalls einige Einheiten der letzten Dezimale von $g$, verändert das Bild also nicht wesentlich.

Das bemerkenswerte Zusammenfallen herzynisch-saxonischer Horstzonen mit den Dichteschwellen scheint die Möglichkeit zu eröffnen, die Schweremessungen mittels Pendelbeobachtungen in Norddeutschland für die Erforschung der dortigen Tektonik und damit auch für die Frage der Erschließung von produktivem Karbon im unbekannten Gebiet zwischen dem westfälischen und dem oberschlesischen Kohlenrevier zu verwerten (Kossmat, Abh. Sächs. Akad., XXXVIII, 2. 1920. S. 57).

Auch die Eörvössche Drehwage wird sich als geeignetes Mittel erweisen, die geophysikalische Erforschung des Untergrundes zu vertiefen. Die Drehwage reagiert ziemlich empfindlich auf seicht liegende Unregelmäßigkeiten in der Massenverteilung, wie sie durch das Auftreten leichterer oder schwererer Gesteinskörper hervorgerufen werden. 
F. Kossmat - Die Bezieh. zwischen Schwereanomalien u. Bau d. Erdrinde. 175

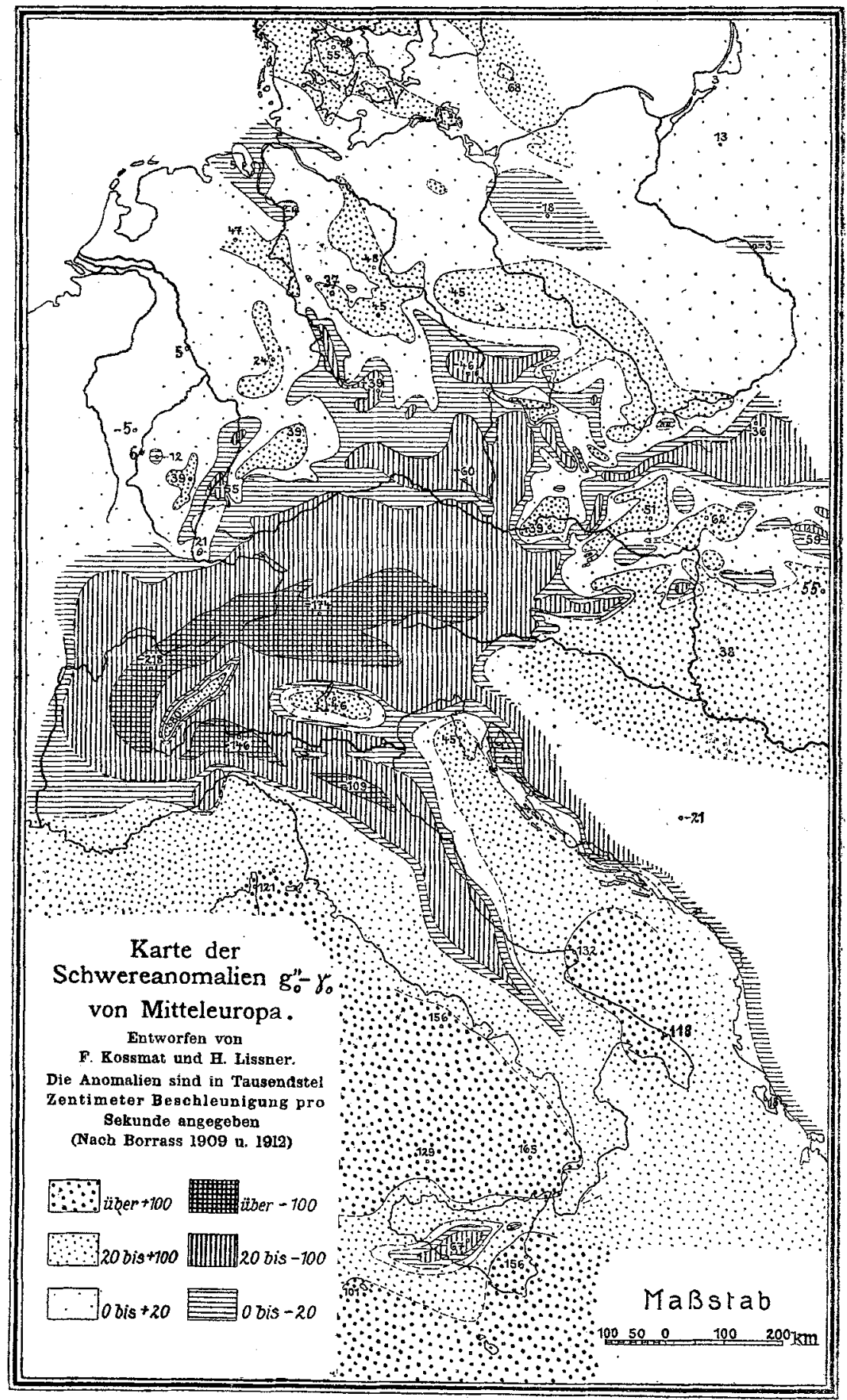

Fig. 2. 
Dies haben die Untersuchungen SoHWEYDars über einen norddeutschen Salzhorst deutlich gezeigt. Die Anwendung dieser Methode ist geeignet, die Pendelbeobachtungen nach bestimmten Richtungen zu ergänzen (aber nicht $\mathrm{zu}$ ersetzen!) und die Deutung der $\Delta g^{\prime \prime}$ zu verfeinern.

Wenig klar sind die Beziehungen zwischen Schwerestörungen und den erdmagnetischen Anomalien, da letztere häufig von akzessorischen Eigenschaften der Gesteine und von anderen Erscheinungen, die mit den großen Massenstörungen nichts zu tun haben, abhängen. Während W. DEEcke für die Adriatische Region gewisse Beziehungen zwischen Schwereanomalien und Erdmagnetismus hervorheben konnte, zeigen die Pendelbeobachtungen HaAsemanns in Ostpreußen keinerlei Anklänge an die magnetische Isanomalenkarte, die TorNquist in den Sitzungsberichten der preuß. Akad. d. Wiss. 1911, S. 834 veröffentlichte. In neuerer Zeit sind von ScHuH magnetische Messungen in Mecklenburg (Mitt. Meckl. Geol. Landesanstalt, XXXII. Rostock 1920), von GöLtNITZ in Sachsen (Beilageband zum Jahrb. f. d. Berg- und Hüttenwesen Sachsens f. 1919) vorgenommen worden. Thre geologische Verwertung ist noch eine schwierige Aufgabe.

\section{Außereuropäisch $\theta$ Schwerebeobachtungen.}

\section{A. Asion.}

1. Im Kaukasus, in Turkestan und im Pamir sind von russischen Geodäten sehr zahlreiche relative Schweremessungen systematisch durchgeführt worden. Sie ergaben im Kaukasus ähnliche Resultate wie in den Alpen, sowohl was das Massendefizit unter dem Gebirge, wie auch unter dem Vorland anbelangt. Leider fehlen entsprechende Messungen für das Innere Kleinasiens, wo im Bereiche der Senken Massenüberschüisse ähnlich denen der pannonischen Ebene zu erwarten sind.

Bedeutende Massendefizite und auch viele absolute Beschleunigungsdefekte haben sich im Pamir und in Turkestan ergeben, z. B.:

\begin{tabular}{c|c|c|c|c|c|c|c}
\hline & $\begin{array}{c}\text { Pamirski } \\
\text { Post }\end{array}$ & $\begin{array}{c}\text { Mittel von } \\
\text { 3 Hoch- } \\
\text { stationen } \\
\text { in Ferghana }\end{array}$ & $\begin{array}{c}\text { Narynsk } \\
\text { S. v. Issyk- } \\
\text { kkul }\end{array}$ & Kokan & $\begin{array}{c}\text { Osch u. } \\
\text { Andidjan }\end{array}$ & Taschkent & Lepsingk \\
\hline Seehöhe & $3700 \mathrm{~m}$ & $4087 \mathrm{~m}$ & $2033 \mathrm{~m}$ & $437 \mathrm{~m}$ & $776 \mathrm{~m}$ & $478 \mathrm{~m}$ & $980 \mathrm{~m}$ \\
\hline$g_{0}-\gamma_{0}=\Delta g$ & -29 & +98 & -67 & -162 & -145 & -49 & -29 \\
\hline$g_{0}^{\prime \prime}-\gamma_{0}=\Delta g^{\prime \prime}$ & -440 & -356 & -290 & -210 & -231 & -103 & -138
\end{tabular}

$A g$ und $\Delta g^{\prime \prime}$ sind in tausendstel $\mathrm{cm}$ Beschleunigung angegeben; $\Delta g^{\prime \prime}$ unter Annahme von 2.7 Gesteindichte gerechnet.

Auffallend groß sind die absoluten Beschleunigungsdefekte in den Niederungen Turkestans. Sie sind nur zum Teil auf die der dortigen 
Schwere entgegenwirkende Anziehung durch Gebirgsheraushebung (bei gleichzeitigem Untergrunddefizit) in der näheren und ferneren Nachbarschaft zu setzen. Der Restbetrag dieser negativen $\Delta g$ hat hier zweifellos ähnliche Bedeutung wie in Vorlandzonen der mediterranen Gebirge Europas, die einen Teil der isostatischen Kompensation der Faltenketten übernehmen, also an ihrer Last tragen helfen.

2. Japan. Die Schweremessungen auf Kiushiu, Shikoku und Hondo zeigen, daß die Massendefizite $\left(-\Delta g^{\prime \prime}\right)$ an die dem Japanischen Meere genäherte Seite der Inseln gerückt sind, während vom pazifischen Ozean her die positiven Beträge in das Land eingreifen. Hierin kommt in der Hauptsache die Erscheinung zum Ausdruck, daß die Massenüberschüsse des benachbarten Tiefseebodens ihre Wirkung bis in die angrenzenden Randgebirge geltend machen (kontinentale Randstörungen). Doch sind im östlichen Hondo große, bis über 100 Anomalieeinheiten steigende Massenüberschüsse vorhanden, deren Betrag über solche Einwirkung hinausgeht und anzeigt, daß schweres Tiefenmagma in diesen Inselgirlanden am Rande der pazifischen Tiefen hoch emporgedrückt sein muß. Die kalifornischen Küstenketten zeigen auffallenderweise diese Erscheinungen nicht. E\& wäre von großem Interesse für die Tektonik, wenn noch mehr Beobachtungen über die Schwereverhältnisse in den ozeanischen Randketten vorliegen würden; vor allem könnte der geologisch gut erforschte ostindische Archipel und die Andenregion Südamerikas sehr interessante Ergebnisse liefern.

3. Fin klassisches Gebiet der Schwereanomalien ist Indien, von wo aus die Pratrsche Isostasietheorie ihren Ausgang nahm. Eine große Rolle spielen in den indischen geodätischen Untersuchungen die Beobachtungen über die meridionale Komponente der Lotablenkungen ${ }^{1}$ ). Sie wird bestimmt durch die Differenz A-G, wobei A die astronomische, von der Lotrichtung bzw. der Libellenlage abhängige Breitenbestimmung bedeutet, $\mathrm{G}$ die aus dem Triangulationsnetz ermittelte geodätische Breite ist. Negatives Vorzeichnen der erhaltenen Differenz bedeutet nördliche Ablenkung, positives südliche. Die Messungen ergaben das bekannte, ursprünglich sehr überraschende Resultat, daß der Himalaya bei weitem nicht die Anziehung auf das Lot ausübt, die seiner sichtbaren Gebirgsmasse entsprechen würde, wenn diese unkompensiert wäre. Die Station Jalpaiguri vor dem östlichen Himalaya sollte z. B. $77^{\prime \prime}$ nördliche Ablenkung zeigen und weist nur $1^{\prime \prime}$ auf. Ebenso zeigt Kaliana nur 1" statt 58". Es -muß also weitgehende Kompensation des Gebirges durch relativ geringere Krustendichte in seinem Untergrund vorhanden sein. Aber außerdem vollzieht sich die Abnahme der nördlichen Ablenkung mit der Entfernung vom Gebirgsfuß zu rasch, als daß sie dadurch allein restlos erklärbar wäre. Nimkar, weniger als 100 Meilen südlich des gestellt.

1) Die Ost-Westkomponente ist erst für eine geringe Zahl von Stationen fest- 
Gebirges, zeigt sogar $5^{\prime \prime}$ südliche Ablenkung. Es wird die Annahme eines unterirdischen Gürtels größerer Dichte verlangt, der südlich der Gangesebene durchzieht und in den Vorlandstationen des Himalaya der Anziehung durch das Hochgebirge entgegenwirkt. Tatsächlich wird diese unterirdische Schwelle durch die Pendelbeobachtungen bestätigt.

Crostwaith rechnete die N-S-Komponente der theoretischen Lotablenkungen für die indischen Stationen aus, und zwar bei Annahme einer vollen Kompensation des Reliefs bis zu einer einheitlichen Ausgleichstiefe von $113,7 \mathrm{~km}$ (die frühere HAYFORD sche Zahl, die später auf 122,2 abgeändert wurde). Es ergab sich dabei keine befriedigende Annäherung an die Beobachtungswerte, so daß entweder die Ausgleichstiefe großen Schwankungen unterworfen sein muß oder die isostatische Kompensation nur wenig weit vorgeschritten ist. Wir haben aus den Verhältnissen im europäischen Mediterrangebiet schließen können, daß letzteres für junge Kettengebirge und ihre Nachbarschaft die Regel ist.

HAYDEN hat sich der Mühe unterzogen, die indischen Lotablenkungen rechnerisch unter der Annahme zu überprüfen, daß die Kompensationstiefe für verschiedene Teile des Reichs Schwankungen unterliege. Er findet, daß an vier Querprofilen vom Himalaya bis zu einer Station im Gangesalluvium (Musoorie-Kaliana, Kurseong-Siliguri, Birond-Pathardi, Murree-Ranjitgarh) der errechnete Wert die größte Annäherung an die beobachteten Verhältnisse zeigt, wenn man für den Himalaya etwa $330 \mathrm{~km}$ Kompensationstiefe, für die Vorlandstation hingegen nur $113,7 \mathrm{~km}$ annimmt. Im großen Durchschnitt werden die Anomalien in der Ebene südlich des Himalaya ("Zentral-Region « der geodät. Provinzen CrostwaITHs) um so mehr verkleinert, je geringer man die Kompensationstiefe nimmt. Die kleinste Unstimmigkeit wird sogar erreicht, wenn man die Kompensation bereits in der Tiefe 0 annimmt, also eine unmögliche Voraussetzung. Dieser Umstand weist nach meiner Ansicht wieder darauf hin, daß keine der beiden Regionen für sich allein kompensiert ist, sondern daß elastische Gewichtsübertragung vom Hochgebirge auf das Vorland stattfindet, wie man sie auch für die Alpen, Karpathen usw. annehmen muß.

Nach einer Uberprüfung durch Lenox-Conyngham leidet die Rechnung HaYDEns übrigens an einem methodischen Fehler, da die von ihm verwendete Formel CrostwartHs auf der Annahme gleichmäßiger Kompensationstiefe innerhalb einer "geodätischen Provinz" beruht, so daB es nicht angeht, für einzelne Stationen einer solchen willkürlich verschiedene Werte einzusetzen. An sich ist aber gegen die Voraussetzung von Schwankungen der Kompensationstiefe nichts einzuwenden.

Die Pendelbeobachtungen im Himalaya mit Vorland haben Ergebnisse gebracht, die in ihren Hauptzügen ausgezeichnet zu den Erfahrungen in Europa stimmen. 
F. Kossmat - Die Bezieh. zwischen Schwereanomalien u. Bau d. Erdrinde. 179

\begin{tabular}{c|c|c|c|c|c|c|c|c|c|c|c|}
\hline & Sandakphu & Moré & Musoorie & Darjeeling & Kurseong & $\begin{array}{c}\text { Dehra } \\
\text { Dun }\end{array}$ & Kaliana & Siliguri Jalpaiguri \\
\hline Meereshöhe & $3586 \mathrm{~m}$ & $4696 \mathrm{~m}$ & $2142 \mathrm{~m}$ & $2123 \mathrm{~m}$ & $1497 \mathrm{~m}$ & $683 \mathrm{~m}$ & $247 \mathrm{~m}$ & $118 \mathrm{~m}$ & $82 \mathrm{~m}$ \\
\hline$g_{0}-y_{0}=\Delta g$ & +198 & $+33 ?$ & +90 & +61 & +5 & -70 & -64 & -147 & -111 \\
\hline$y_{0}^{\prime}-y_{0}=\Delta g^{\prime \prime}$ & -155 & $-506 ?$ & -132 & -153 & -146 & -136 & -93 & -155 & -117
\end{tabular}

Für die Station Moré liegen nur die alten Messungen von Basevi vor, deren Verwertung wegen der Schwierigkeit, gewisse instrumentelle Beobachtungsfehler

(Mitschwingen des Stativs) nachträglich zu ermitteln, nicht einwandfrei ist.

Das durch Lot und Pendel nachgewiesene Vorlanddefizit erklärte BURrard, der Leiter der Geodätischen Aufnahmen Indiens, durch eine etwa 20 englische Meilen tief hinabreichende, mit Alluvionen erfüllte Spalte, die durch Zerrung der Unterkruste (Tensionsschale) beim Schrumpfen über einem Erdkern von mehr konstantem Volumen entstanden sei. Gegen diese Annahme, die weder mit der Plastizität der tieferen Erdschalen, noch mit der Tektonik des Himalaya in Einklang zu bringen ist, wendeten sich HaYdeN und OLdHAM. Beide erklären, wie es ja nach den geologischen Verhältnissen kaum anders denkbar ist, das Vorland als eine mit jungen Ablagerungen erfüllte Randmulde. OLDHAM machte sogar den Versuch, auf Grund der Pendelbeobachtungen die Tiefe der jungen Ablagerungen, deren Dichte er mit 2,16 gegen 2,65 der alten Krustengesteine annimmt, zu rechnen, und er glaubt, gute Utbereinstimmung zwischen dem so ermittelten geodätischen Defizit-Wert und der Wahrscheinlichkeitsziffer von 15 000-20000 Fuß Mächtigkeit für Quartär bis Pliozän zu erkennen. Seine Rechnungen basieren auf einer theoretisch vereinfachten Gestalt des kompensiert gedachten Himalaya. Sie werden von BURRARD sowohl aus diesem Grunde, wie auch wegen der zu geringen Durchschnittsdichte, die für das Vorlandsediment zugrunde gelegt ist, bemängelt. Mit Recht wird von ihm auch hervorgehoben, daß die Defizite nicht nur auf der Mächtigkeit der leichten Oberflächengesteine, sondern auch auf der Dichteverteilung in den tieferen Rindenteilen beruhen, so daß ein weiterer unbekannter Faktor eintritt, der nachweislich an wichtigen Stellen der gangetischen Ebene zu ganz abweichenden und sogar entgegengesetzten Resultaten führt. Wichtig ist aber, daß negative Schwerewerte vorkommen, die bei Zugrundelegung eines Sedimenttrogs von $20000 \mathrm{FuB}$ Tiefe und einer wahrscheinlichen Dichte von 2,4 (statt 2,16 bei Oldham) noch außerdem ein Massendefizit in der tieferen Erdschale verlangen. Diese Erscheinung weist nach meiner Ansicht wieder auf unterirdische Massenverschiebungen in der plastischen Region, in diesem Falle also auf eine Verstärkung der salischen Magmazone unter dem Faltengebirge und Vorland, wie sie auch für die europäischen Kettengebirge anzunehmen ist. (Vgl. das schematische Profil eines Faltengebirges in Abh. d. Sächs. Akad., Bd. XXXVIII, 2, S. 47.) 


\section{B. Deutsch-Ostafrika.}

Großen grundsätzlichen Wert haben die Beobachtungen von KorssCHǗTER, die in den Jahren 1899/1900 vorgenommen wurden. Nachdem er von den Werten $g_{0}$ und $g_{0}^{\prime \prime}$ den Normalwert $\gamma_{0}$, vermehrt um die Korrektion $+\delta \gamma$ für die Ozeaneinwirkung, abgezogen hat, kommt er zu dem Ergebnis, daß die ausgedehnten Plateaus im großen und ganzen kompensiert sind, und zwar sind die Massendefizite ( $\left.-A g^{\prime \prime}\right)$ im Innern des Kontinents auch nach dieser Korrektion noch immer viel größer als in der küstennahen Zone. Sie betragen für erstere im Mittel -142 Einheiten entsprechend einem Kontinentalplateau von $1245 \mathrm{~m}$ mittlerer Föhe und 2,76 spezifischem Gewicht. In der Küstenzone, von $350 \mathrm{~km}$ Meeresabstand bis zum Kontinentalrand gerechnet, ist der Durchschnitt nur -16 Einheiten entsprechend $140 \mathrm{~m}$ mittlerer Höhe. Es ist hierin eine Annäherung an die ozeanischen Dichteverhältnisse exkennbar. Diese Defizite sind für die mittlere Höhe Deutsch-Ostafrikas etwas zu klein, so daß eine Überschwere dieses Landes um einige Einheiten (etwa 5) vorhanden zu sein scheint, vorausgesetzt, daß HELMERTs Normalwert der Schwere $\left(\gamma_{0}\right)$ nicht um diesen Betrag zu klein ist. Für die Einzelheiten des Reliefs besteht nach den sorgfältigen Rechnungen KoHLschüTrERs keine Kompensation - weder für die einzelnen Gebirgserhebungen noch für die großen Bruchgräben. Letztere weisen beträchtliche ungedeckte Defizite auf.

\begin{tabular}{c|c|c|c|c|c|c}
\hline & \multicolumn{2}{|c|}{$\begin{array}{c}\text { Küstenferne Plateau- } \\
\text { stationen }\end{array}$} & $\begin{array}{c}\text { Küstennahe } \\
\text { Plateau- } \\
\text { station } \\
\text { Wilhelmstal }\end{array}$ & $\begin{array}{c}\text { Küstenferne } \\
\text { Graben- } \\
\text { station } \\
\text { Moliro }\end{array}$ & $\begin{array}{c}\text { Küstennahe } \\
\text { Graben- } \\
\text { station } \\
\text { Rufu }\end{array}$ & Daressallam \\
\cline { 2 - 7 } & Isimia & Ndjilla & \\
\hline Meereshöhe & $1733 \mathrm{~m}$ & $1242 \mathrm{~m}$ & $1378 \mathrm{~m}$ & $792 \mathrm{~m}$ & $656 \mathrm{~m}$ & $7 \mathrm{~m}$ \\
\hline$g_{0}-\left(\gamma_{0}+\delta \gamma\right)$ & +69 & +2 & +79 & -61 & -39 & -23 \\
\hline \begin{tabular}{c}
$g_{0}^{\prime \prime}-\left(\gamma_{0}+\delta \gamma\right)$ \\
\hline $\begin{array}{c}\delta=\text { Konti- } \\
\text { nental-Rand- } \\
\text { störung }\end{array}$
\end{tabular} & -130 & -140 & -79 & -153 & -116 & -24 \\
\hline $\begin{array}{c}\text { Entfernung } \\
\text { der Station } \\
\text { vonder Tiefsee }\end{array}$ & $880 \mathrm{~km}$ & $750 \mathrm{~km}$ & $140 \mathrm{~km}$ & $960 \mathrm{~km}$ & $240 \mathrm{~km}$ & $40 \mathrm{~km}$ \\
\hline
\end{tabular}

Die Gräben sind offenkundige Zerrungsspalten der Erdrinde, in die leichtes Material der beiden Ränder hineingesackt ist'1). Die Schwere-

1) Vgl. auch Wegrarer, Entstehung der Kontinente und Ozeane. 2. Aufl. 1920. S. 38. Eine nähere Untersuchung der geologischen Beziehungen wird eine in Druck befindliche Arbeit von E. KRENKEL über Brüche, Erdbeben und Schwerestörungen in Deutsch-Ostafrika bringen. 
verhältnisse ändern sich aber dort, wo basisches Magma von großer Dichte in größeren Massen hochgestiegen ist. Dies zeigen die bekannten Messungen der österreichischen Rote Meer-Expedition mit der Fregatte "Pola " und die Heckrrschen Beobachtungen im Golf von Aden. Hier herrschen naturgemäß große Massenüberschüsse.

KoHLschürter hat zum Vergleich auf Grund von HeCkERs Beobachtungen auch die Ergebnisse für die Ozeanstrecke Sidney-NeuseelandSamoa gerechnet und kommt zu ähnlichen grundsätzlichen Ergebnissen wie für Kontinentgebjete. Es herrscht Kompensation für ein bestimmtes mittleres Niveau, das in dem genannten Ozeanabschnitt in $-5000 \mathrm{~m}$ liegt, hingegen sind die Abweichungen davon nicht kompensiert. Der unterseeische Tongarücken hat großen Schwereüberschuß, dafür aber der als Vortiefe zu bezeichnende Tongagraben ein absolutes Schweredefizit. Hier haben wir Beziehungen, wie sie zwischen Kettengebirge und Vorland herrschen. Die einzelnen Teile des Ozeanbodens zeigen somit untereinander ähnliches Verhalten wie jene der Kontinentalregion, nur die absolute Dichte der Unterlage ist im ganzen genommen größer.

\section{Nordamerika.}

Die Deutung der Schwereanomalien hat in Nordamerika etwas andere Wege eingeschlagen als in Europa, da bei den dort angewandten Methoden die Pratrsche Isostasietheorie stark in den Vordergrund trat.

Wie in der Einleitung erwähnt wurde, ist die totale Schwerestörung $g_{0}-\gamma_{0}$ von der zufälligen Höhenlage des Beobachtungsortes gegenüber dem mittleren Niveau der weiteren Umgebung abhängig. Sie verschiebt sich nach der positiven Seite, wenn ersterer sich höher emporhebt, und nach der negativen, wenn er in einem tieferen Einschnitt liegt. Abgesehen davon müssen auf einer Erde mit ungleichmäßiger Massenverteilung die Beschleunigungswerte auch bei Bestehen voller Isostasie noch Schwereanomalien aufweisen. Wenn z. B. ein höher aufragender Erdsektor von geringerer Dichte in Gleichgewicht steht mit einem benachbarten niedrigeren von entsprechend größerer Dichte, so wird ein auf letzterem liegender Beobachtungsort eine unternormale Beschleunigung aufweisen, weil diese durch die angrenzende Massenheraushebung in negativem Sinne beeinflußt wird. Das Umgekehrte wird auf der Oberfläche des hochliegenden Sektors eintreten. Dies zeigt sich am besten an der schon S. 168 erwähnten Kontinentalrandstörung.

Die amerikanischen Geophysiker HAYFORD und BowIE gingen daher nicht von $g_{0}-\gamma_{0}$ aus, sondern stellten dem unmittelbar beobachteten Beschleunigungswert $g$ jenen gegenüber, den man erhält, wenn man den Normalwert $\gamma_{0}$ auf die Seehöhe des Ortes umrechnet und an dem so erhaltenen $\gamma$ alle jene Reduktionen anbringt, die sich bei Berïck- 
sichtigung des gesamten Erdreliefs unter Voraussetzung der Isostasie ergeben. Da diese Einflüsse von der Tiefe abhängen, bis zu der die UngleichmäBigkeiten der Massenverteilung reichen, muß ein wahrscheinlicher Wert für erstere ermittelt werden. Als solchen legte HaYFord seinen Rechnungen eine einheitliche Kompensationstiefe von $113,7 \mathrm{~km}$ zugrunde. Er verbesserte diese Ziffer später auf $122,2 \mathrm{~km}$, - was dem von HelmerT auf Grund der Schwereverhältnisse am Kontinentalrand gegebenen Wert $118 \mathrm{~km}$ sehr nahe kommt - doch konnte diese kleine Veränderung für die Ausrechnung der Anomalien nicht mehr verwendet werden.

$g$ beobachtet minus $g$ berechnet (unter Voraussetzung der isostatischen Reliefkompensation innerhalb einer bestimmten Tiefe) stellt somit die Haxfordsche Schwereanomalie ${ }^{1}$ ) dar, die meist kurz als $g-g_{c}$ bezeichnet wird. Sie bedentet eine wichtige Neuerung auf dem Gebiete der Reduktionsmethoden, da sie vom Relief unabhängig gemacht ist und daher für die physische Erdoberfläche unmittelbar vergleichbare Werte liefert.

Die so errechneten Anomalien ("residuals 《) sind als Isostasiestörungen aufzufassen, wenn die gemachten Voraussetzungen gelten: nämlich gleichmäßige Massenschichtung unterhalb einer einheitlichen Kompensationstiefe $(113,7 \mathrm{~km})$ und gleichmäßige vertikale Verteilung der über dieser liegenden, das Relief kompensierenden Massenüberschüsse und Defizite. Es ist selbstverständlich, daß auch auf diesem Wege nur eine Annäherung an die völlige Deutung der Anomalien herbeigeführt wird.

Die Überarbeitung von 124 Pendelstationen der Vereinigten Staaten ergab das sehr bemerkenswerte Resultat, daß unter Anwendung dieser sisostatischen " Reduktionsmethode die verbleibenden Anomalien im Mittel nur mehr 20 Einheiten der letzten Dezimale von $g\left(0,020 \mathrm{~cm} / \mathrm{sec}^{2}\right)$ ohne Rücksicht auf das Vorzeichen ausmachen, $d . h$. daß die regionale Schwankung des Schwerewertes innerhalb eines sehr engen Spielraumes liegt, der durch eine Gesteinplatte von $200 \mathrm{~m}$ Höhe und 2,4 Dichte darstellbar ist. Zu einem ähnlichen, sogar noch günstigeren Ergebnis führte die Anwendung dieser Methode auf die Reduktion der beobachteten Lotablenkungen in den Vereinigten Staaten. Diese Tatsache spricht für weitgehende Isostasie; und zwar geht die Ansicht der ge-

1) Die Definition lautet: "The computed value of gravity at the station, $g_{r}$, is the theoretical value of gravity at sea level, $\gamma_{0}$, corrected for elevation and for topography and compensation. The column $g-g_{c}$ therefore represents the departures of the observed values from computed values based on the HrLMers formula of 1901, upon the usual reducton for elevation and upon the new method reductions that take account of topography and compensation." (A. Bowre: Effect of topography and compensation upon the intensity of gravity. Second paper. U.St. Coast and geodetic Survey. Special Publication Nr. 12. Washington 1912. S. 8. 
nannten amerikanischen Forscher dahin, daß eine solche durchschnittlich schon für relativ kleine Reliefelemente von $18,8 \mathrm{~km}$ Radius Geltung hat. Eine elastische Ubertragung der Kompensation auf Nachbargebiete wird nicht angenommen ${ }^{1}$ ).

Während HAYFORD und BowIE die verbleibenden Anomalien als kleine Unvollkommenheiten der Isostasie gelten lassen, suchte GILBERT die Abweichungen von Pratrs Theorie noch weiter einzuschränken, indem er die Voraussetzungen der Berechnung modifizierte:

1. Durch vertikale Verschiedenheiten in der Massenanordnung. Liegen in zwei einander das Gleichgewicht haltenden Erdsektoren die dichteren Massen e:nmal oben, ein anderes Mal unten, so ist im ersteren Falle die Beschleunigung an der Oberfläche etwas vergrößert, so daß eine positive $A b w e i c h u n g$ von der Isostasie vorgetäuscht wird, et viz. Die für Erklärung der größten Anomalien verlangten Umschichtungen sind allerdings so enorm, daß sie den Erfahrungen über den Bau der Erdkruste widersprechen.

2. Durch verschiedene Kompensationstiefe. Nimmt man z. B. unter einer Erhebungsregion eine kleinere Kompensationstiefe an als die von HAYFORD zugrunde gelegten $113,7 \mathrm{~km}$, so sinkt der berechnete Wert $g_{c}$, es verschiebt sich also die Differenz $g-g_{c}$ nach der positiven Seite; bei Zunahme der Kompensationstiefe tritt das entgegengesetzte Verhalten ein. Aber auch bei dieser Annahme bleiben größere Anomalien, wie die oft enge benachbarten Überschüsse und Defizite, die wir $\mathfrak{u}$. a. auch in niedrigem Gelände von geringer Meereshöhe (vgl. Dänemark, NW.-Deutschland usw.) finden, unerklärt.

3. Durch primäre Heterogenität des Erdkerns. Diese Annahme entzieht sich völlig der Kontrolle. Entgegen der Ansicht GIIBERTS scheint sie mir in den Hintergrund zu treten gegenüber den offenkundigen großen Massenstörungen in der Rinde, deren engen Zusammenhang mit tektonischen Grundzügen man in klarer Weise aus den Schwereanomalien der Kategorie $g_{0}^{\prime \prime}-\gamma_{0}$ ablesen kann.

Die Karte der HAYFoRDschen Anomalien $g-g_{c}$ zeigt ähnlich wie eine solche der absoluten Schwereanomalien wenig deutliche Beziehungen zu geologischen Verhältnissen, was auch G. K. GILBERT u. a. betonen. Wir sehen in ihr z. B. regionalen Schwereüberschuß (bis +52 Einheiten für die Black Hills) in den Rocky Mountains, aber ebenso gut in Teilen der Ebenen (bis +52 Einheiten in St. Paul); wir finden ein ausgedehntes

1) Diese weitgehende Auffassung steht nicht in Einklang mit den Ergebnissen HoLMERTs, nach denen die Isostasie selbst für Reliefelemente bis auf einige hundert Kilometer lineare Ausdehnung nicht streng zutrifft. Die Verhältnisse in Europa, wo so ausgedehnte, auch große Teile von Rußland kennzeichnende Massenüberschüsse vorhanden sind, sprechen entschieden nur für regionale Kompensation. Thenso läßt sich das so interessante geologische und grayimetrische Verhalten der Randsenken (ihr passives Hinabziehen) nach meiner Ansicht nur durch Übertragung eines Teils der Kompensation auf die Nachbarschaft erklären. 
Schweredefizit in dem Sierra Nevada-System ${ }^{1}$ ) und in den Prairien, wo das Maximum mit -52 Einheiten bei Mena in Westarkansas vermerkt wird. Immerhin sind einige, vom orogenetischen Gesichtspunkt wichtige Erscheinungen zu beobachten: Östlich von dem positiven $g-g_{c}$ der Rocky Mountains zieht in deren östlichem Vorland ein Defizitgürtel von Minnesota über Nebrasca nach Neu Mexiko und Texas, während noch weiter östlich eine auffällige Schwelle größerer Schwere vom westlichen Lake Superior über St. Paul in Minn., Lincoln in Nebr., Ellsworth in Kans. nach Jerico in Texas verläuft, um dann näher dem Mississippigebiet einem neuen breiten Defizitgürtel Platz zu machen, dessen Maximum bei dem schon genannten Orte Mena in Arkansas liegt. Vielleicht prägen sich hierin einige der unterirdischen, vom Faltengürtel ausgehenden Massenverschiebungen aus, von denen im ersten Abschnitt die Rede war.

Daß man aus den HaYForDschen Kurven nichts über die Bewegungstendenz eines Gebiets herauslesen kann, hängt damit zusammen, daß die orogenetischen Kräfte und die Trägheit der Erdrinde eine vollständige Einstellung auf den isostatischen Zustand nicht zulassen. Als einen Mangel im Kartenbilde empfindet man, daß letzteres nichts darüber aussagt, ob ein Schwereplus auf einem auch unterirdischen Massenüberschuß oder nur auf einem zu kleinen Massendefizit unter einem Gebirge beruht; gerade dieser Umstand hat für den Geologen, der die unterirdischen Massenbewegungen bei der Gebirgsbildung verfolgen will, mehr Interesse als die erfolgte wirkliche Schwerestörung.

Für diese Fragen eignet sich mehr die Bouguersche Reduktionsmethode, die uns für Mittel- und Nordeuropa so wichtige Aufschlïsse über das Krustenverhalten bringt, da sie - wenn auch nicht rein ein Bild der Dichteverteilung in dem unterhalb einer bestimmten Niveaufläche (Meeresspiegel) gelegenen Teile der Erdrinde vermittelt. Daß gewisse systematische Einflüsse, wie die Kontinentalrandstörung, bei der Deutung Berücksichtigung finden sollen, wurde bereits in der Einleitung S. 168 hervorgehoben. Doch hat es seine Vorteile, zunächst die $\Delta g^{\prime \prime}$ in ihrer ursprünglichen Form zu geben, weil sie gerade in dieser geeignet sind, eine Kritik der anzubringenden Verbesserungen zu ermöglichen. Auch ist es für gewisse Fragen nicht ausschlaggebend, ob eine Massenstörung mehr oder minder unmittelbar unter dem Beobachtungsort anzunehmen ist oder nur in seinem Bereich.

Manche Probleme dieser Art lassen sich nur durch Vergleiche lösen. So könnte man z. B. in Europa die Massenüberschüsse rund um das tiefe tyrrhenische Meeresbecken zu einem großen Teil auf Rechnung

1) Daß HAYjonds Karte für die pazifischen Randgebirge (Sierra Nevadasystem) und für den gröBten Teil des atlantischen Saumes negative $g-g_{c}$ zeigt, ist recht eigentümlich. Ws scheint mir dieser Umstand die Vermutung nahe zu legen, daß in seiner Berechnung für die ozeanische Steilrandwirkung doch ein zu großer Betrag abgezogen wurde. 
des letzteren setzen (als Kontinentalrandstörung), wenn nicht ganz analoge Verhältnisse bei wesentlich geringeren Höhenunterschieden auch in der Umwallung der ungarischen Ebene auftreten und damit zeigen würden, daß eine unterirdische Massenverschiebung von diesen Innensenken gegen ihre Umrandung tatsächlich eingetreten sein muß (S. 172).

Leider reichen die 124 Schwerestationen der Vereinigten Staaten nicht aus, um die $\Delta g^{\prime \prime}$ so in Zusammenhang mit den tektonischen Elementen zu betrachten, wie dies bei dem dichten Beobachtungsnetz Mitteleuropas möglich war. In den Hauptzügen läßt sich aus ihnen nur feststellen, da $B$ unter den jungen Faltengebirgen des amerikanischen Westens große Massendefizite (z. B. Gennison in Col. -229) auftreten, die auch in das angrenzende Prairiengebiet hineinreichen, während eine Schwelle relativ größerer Dichte vom westlichen Teile des Lake Superior in SW-Richtung durch Ostnebraska und Kansas nach Oklahoma und Texas zieht, also in Beziehung zu dem betreffenden Zug auf der HAYFoRDschen Anomaliekarte steht. Die Appalachen weisen kompensierende Defizite auf, die mit jenen des östlichen Prairiengebiets verschwimmen und nicht mehr dem alten Faltenbau des Untergrundes in jener Weise angepaBt sind, wie in den jungen Faltengebirgen Europas. Daß schließlich unter dem atlantischen und pazifischen Ozean schon nahe dem Rand größere Krustendichte herrschen muß als durchschnittlich unter dem Kontinent, wird durch das besonders in Kalifornien ziemlich rasch erfolgende Ansteigen der Massenstörungskurven sehr kiar zum Ausdruck gebracht.

\section{Die Frage der Faltungsursachen.}

Für die Kettengebirge ist die Form langer bis endloser Bänder kennzeichnend, die sich zwischen starreren Regionen der Erdrinde durchwinden, große Girlanden oder Schleifen beschreiben und sich an Hindernissen stauen, so daß sie bald als eng zusammengeraffte Stränge vom Alpentypus auftreten, bald weit auseinander streben und ausgedehnte Innensenken einschließen oder auch rutenförmig sich verästeln, wie die großen Virgationen Turkestans. Eine derartige Erscheinungsform erinnert an träges $\mathbb{F l i e ß e n , ~ d a s ~ a b e r ~ n i c h t ~ e t w a ~ d u r c h ~ A b s t r o ̈ m e n ~ v o n ~}$ Hebungsachsen veranlabi ist, sondern ausgedehnte tangentiale Krustenbewegungen zum Ausdruck bringt. Auch O. AMPFERER, der in seiner bekannten Unterströmungstheorie die Faltengebirge ursprünglich als Streifen eigener Entstehung auffaßte, in denen die Bewegung des plastischen Untergrundes durch Schaffen eines Vertikalgefälles erklärt wurde, hat in einer kürzlich erschienenen Veröffentlichung: "Geometrische Erwägungen über den Bau der Alpen «, Mitteilungen der Geolog. Ges. Wien 1919/20, S. 135, seine frühere Ansicht aufgegeben und nimmt eine weitausgedehnte tangentiale Rindentrift an. 
Besonders wichtig erscheint mir in diesem Zusammenhange auch das Auftreten der einen beträchtlichen Teil des Erdumfanges durchlaufenden, mehr oder minder quer zu den Breitengraden gerichteten Sprünge, die den indoafrikanischen Kontinentalblock durchschneiden. Die Tatsache, daß diese Brüche bei Annäherung an die Kettengebirge zersplittern und erlöschen, kennzeichnet sie als Gebilde, die in ursächlicher Beziehung zu deren Faltung stehen. Sie wären in ihrem Riesenausmaß und ihrer Gleichförmigkeit unmöglich zu erklären, wenn die von ihnen durchsetzten Schollen der Erdrinde nicht eine Tangentialbewegung ausgeführt hätten, die für den Verlauf der Kettengebirge bestimmend war. Ich habe den Eindruck, daß hier Risse vorliegen, deren vorwiegend meridionaler Verlauf durch Gleiten der oberen Erdschalen auf den inneren Teilen des Erdellipsoids bedingt ist.

Die regionalen Verschiedenheiten der Massenverteilung auf der Erde und die damit in Verbindung stehenden erheblichen Unterschiede in den Schwerpunktlagen scheinen tatsächlich die Ursache langsamer Krustenwanderungen zu sein. Es ergaben sich, da diese Massenverteilungen im Laufe der geologischen Perioden sehr erheblichen Änderungen ausgesetzt waren, ständig neue Impulse, die auf der rotierenden, der Anziehung von Sonne und Mond ausgesetzten Erde eine beträchtliche Tangentialkomponente liefern mußten und das richtunggebende Moment bei den Verschiebungen der äußeren Erdschale darstellen. dürften. Die Kettengebirge und mit ihnen die Geosyklinalen sind stark nachgiebige Zwischenstreifen, die sich dort entwickeln, wo sich weite Rindenfelder nähern, sei es, dab ihre Bewegung entgegengesetzt ist, sei es, daß sie verschiedene Größe besitzt. Auch die Verschiebung der Klimazonen spricht zugunsten tangentialer Krustenverlagerungen, für deren Erklärung die einfache Kontraktionstheorie versagt.

Für den Zusammenschub, der die Faltung hervorruft, kommt als Erklärung sowohl Verkleinerung des Erdumfanges, wie auch Vergrößerung der Rindenoberfläche in Betracht. In neuerer Zeit wird in letzterer Hinsicht, wohl mit Recht, besonderes Gewicht auf das Empordringen von Magmamaterial gelegt, das nach und nach einen sehr großen Prozentsatz der Kontinentalkruste und zweifellos auch des Meeresbodens in Anspruch genommen hat. Nach meiner Ansicht haben aber die Krustenbewegungen nicht den Charakter der von WEGENER angenommenen Schollentrift (WEGENER, Die Entstehung der Ozeane und Kontinente, 2. Aufl., Verlag Vieweg 1920), bei der die Ozeanböden geradezu als freigelegte Oberfläche der Magmasphäre gedeutet werden ${ }^{1}$ ). Besonders die Zusammenhänge der alten und neuen Ketten in der nördlichen Umwallung des Indischen Ozeans lassen, wie ich glaube, für dieses wichtige Gebiet eine Erklärung in derartigem Sinne nicht zu. Die von WEGENEE

1) Vgl. dazu die Veröffentlichung über den von der Berliner Gesellschaft für Erdkunde veranstalteten Vortrag von WEGENER mit anschließender Aussprache. Zeitschr. d. Ges. f. Erdkunde, Berlin 1921. 
u. a. sehr stark hervorgehobene Unmöglichkeit, daß ein Stück Ozeanboden einen abgesunkenen kontinentalen Krustenteil darstellen könne, besteht meines Erachtens nicht. Daß Schwereveränderungen, die zum regionalen Sinken großer Krustenteile führen, tatsächlich erfolgen, zeigen uns deutlich die Erscheinungen in weiten und tiefen Geosynklinalen der mesozoischen Zeit, deren Platz vorher durch ausgedehnte Hochgebiete - man vergleiche das Schicksal der variskischen Gebirge eingenommen war. Der Unterschied zwischen derartigen Erscheinungen und dem Absinken zu ozeanischen Tiefen ist nur ein gradueller und nicht ein prinzipieller. Für das Sinken sind Massenverschiebungen bei der Bildung der großen Kettengürtel wichtig, weil dabei schweres Tiefenmagma verdrängt wird und andere Krustenteile unterströmt. Andauernde Spalteneruptionen in letzteren werden durch Beschleunigung der Entgasuing und Erstarrung in weiter Fläche die Erhöhung der Dichte befördern.

\section{Literaturübersicht.}

Allgemeines.

Borrass, E., Bericht über die relativen Messungen der Schwerkraft mit Pendelapparaten. Zeit von 1908-1909. Verhand. d. XVI. allgem. Konferenz für internat. Erdmessung. Berlin 1912. ПII. T., 288 S. - Zeit von 1909-1912. XVII. allg. Konferenz für internat. Erdmessung. Berlin 1914. II. Teil, $82 \mathrm{~S}$.

Gmbert, G. K., Interpretation of anomalies of gravity. U. S. Geol. Survey. Profess. papers 85 C. Washington 1913. S. 29-36 (mit Angaben der amerikanischen Literatur).

Heumert, F. R., Die Schwerkraft und die Massenverteilung auf der Erde. Enzyklopädie der math. Wissenschaften. Bd. VI, 1. B., Heft 2. Leipzig, Teubner, 1910, mit vielen Literaturangaben.

HeLMERT, F. R., Die Erfahrungsgrundlagen der Lehre vom allgemeinen Gleichgewichtszustand der Massen der Erdkruste. Sitzungsber. d. preuß. Akad. d. Wiss. Berlin 1912. S. 308.

HегмеRт, F. R., Unvollkommenheiten im Gleichgewichtszustande der Erdkruste. Sitzungsber. d. preuß. Akad. d. Wiss. Berlin 1908. S. 1058.

Helmert, F. R., Geoid und Erdellipsoid. Zeitschr. d. Ges. f. Erdkunde. Berlin 1913. S. $17-34$.

HeLmeRT, F. R., Neue Formeln für den Verlauf der Schwerkraft im Meeresniveau beim Festland. Sitzungsber. d. preuß. Akad. 1915. S. 676.

Hzumert, F. R., Bericht über die Tätigkeit des Zentralbureaus der internat. Erdmessung im Jahre 1915. N. F. der Veröff. d. Zentralbureaus f. internat. Erdmessung. Nr. 29. Berlin 1916. S. 6.

KöPPEN, W., Über Isostasie und die Natur der Kontinente. Geograph. Zeitschr. XXV. Bd. Leipzig 1919, 1. Heft. S. 39-48.

Kossmat, F., Die mediterranen Kettengebirge in ihrer Beziehung zum Gleich. gewichtszustande der Erdrinde. Abh. d. Sächs. Akad. d. Wiss. Bd. XXXVIII, 2. Leipzig 1920. S. 1-62, mit Karte.

DE MarohI, L., Teoria elastica delle dislocazione tectoniche. Atti R. Accad. d. Lincei. Rendiconti XV. Tom. 1907. S. 384, 499.

Dr Marcm, L., Teoria elastica dell' isostasi terrestro. ibid. S. 910. 
Mrisswer, O., Isostasie und Küstentypus. Petermanns Mitt. Bd. LXIV. Gotha 1918. S. 221.

MesserschmitT, J. B., Die wichtigsten Beziehungen zwischen der Geodäsie und der Geologie. Jahresber. d. physil. Ges. zu Zürich. Bd. VI. S. 15.

Messergonmitr, J. B., Die Schwerebestimmungen an der Erdoberfläche. Sammlung Wissenschaft, Verlag Vieweg, Heft 27. Braunschweig 1908.

PfafF, Ưber Messungen der Schwerkraft. Beiträge zur Geophysik. Leipzig 1914. XIII.

ScHWEYDAR, W., Die Bedeutung der Drehwage von Eötvös für die geologische Forschung nebst Mitteilung der Ergebnisse einiger Messungen. Zeitschr. f. prakt. Geologie. Berlin 1918. S. 157-162.

ScHWINNER, R., Vulkanismus und Gebirgsbildung. Zeitschr. f. Vulkanologie. 1920. Tams, E., Isostasie und Erdbeben. Zentralbl. f. Min., Geol. u. Pal. Stuttgart 1920.

TAMs, E., Drehwage und Schweremessungen in ihrer Bedeutung für die Geologie. Geol. Rundsch. X. Leipzig 1919. S. 1-13.

TAMs, E., Über die Fortpflanzungsgeschwindigkeit der seismischen Oberflächenwellen längs kontinentaler und ozeanischer Wege. Zentralbl. f. Min., Geol. u. Pal. Stuttgart 1921, Heft 2, 3.

WeGENer, A., Die Entstehung der Kontinente und Ozeane. 2. Aufl. Sammlung Wissensohaft, Heft 66. Verlag Vieweg. Braunschweig 1920.

Zahlreiche Referate über Arbeiten auf dem Gebiet der Schweremessungen finden sich im Geographischen Jahrbuch, herausgeg. von WAGNER, besonders Bd. 30, 1907, Bd. 36, 1913, und in GerLards Beiträgen zur Geophysik.

\section{Europa.}

AMPFERER, O., Über die geologische Deutung von Schwereabweichungen. Verh. d. Geol. Reichsanstalt. Wien 1918. S. 31.

Costanzi, G., Abozzo d'una Carta delle isanomale della gravitá nell' Europa centrale e nel Giappone meridionale. Rivista geographica italiana. XIV. Firenze 1907. p. $364-369$.

DEECKE, W., Erdmagnetismus und Schwere in ihrem Zusammenhang mit dem geologischen Bau von Pommern und dessen Nachbargebieten. Nenes Jahrb. f. Min., Geol., Pal. XXII. Beilagebd. 1906. S. 127.

DeEcke, W., Der geologische Bau der Apenninenhalbinsel und die Schweremessungen. Neues Jahrb. f. Min., Geol. Pal., Festbd. 1907. S. 129-158.

DEEOKE, W., Schweremessungen im südlichen Schwarzwald und in ElsaB-Lothringen. Ber. d. nat. Ges. zu Freiburg i. Br. Bd. XVIII. 1910. S. 57.

HaAsmmanis, L., Bestimmung der Schwerkraft auf 35 Stationen in der Nähe des Meridians $9^{\circ} \mathrm{E}$. v. Gr., ferner in Ostpreußen und in den deutschen Mittel. gebirgen. Veröff. d. preuß. geodät. Inst. Neue Folge 71. Berlin 1916.

HeIm, A., Das Gewicht der Berge. Jahrb. d. Schweizer Alpenklub. Zürich. LIII. Bd. 1918. S. $179 \mathrm{ff}$.

Harm, A., Geologie der Schweiz. II Bd., Heft 2. Leipzig, Tanohnitz, 1919.

NrFTHAMMER, Schwerebestimmungen der Schweizerischen geodätischen Kommission. S. 43-62. Verh. d. Schweiz. naturf. Ges. 91. Sitzungsb. Glarus 1908.

RIcco, A., Anomali della gravitá e del magnetismo terrestre in Calabria e Sicilia in relazione alla costituzione del suolo. Boll. soc. sismolog. Italiana Bd. XII. 1908.

\section{Außereuropäische Länder.}

Bowrs, W., Some relations between gravity anomalies and the geologic formations in the United States. Am. Journ. of Sci. 4th. Ser. Bd. XXXIIT. 1912.

Burrard, S., Geological interpretations of geodetic results: A critical examination of Mr. R. D. OLdHAMs treatise on Himalayan structure. Geograph. Journal. Bd. LII. 1918. p. $237 \mathrm{ff}$. 
Monray Davis A., The Problem of the Himalaya and the Gangetic trough. Geogr. Journal. Bd. LI. 1918. p. 175-182, mit Literatur.

Ecoles, Bericht über Lotablenkungen in Indien. Verh. d. XVII. Konf. f. internat. Erdmessung. Hamburg 1912. I. S. 316.

HAYDEN, H. H., Notes on the relationship of the Himalaya to the Indogangetic Plain and the Indian Peninsula. Records geol. Surv. of India. Vol. XLIII. 2. Calcutta 1913.

HAYFORD, J. F. and W. BowLE, The Effect of topography and isostatic compensation upon the intensity of gravity. U. S. Coast and Geodetic Survey. Spec. Publ. No. 10. Washington 1912.

Bowre, W., The effect of topography etc. Second paper. Ibid. No. 12. 1912.

KoHLschüTter, E., Ửber den Bau der Firdkruste in Deutsch-Ostafrika. Nachrichten von d. kg. Ges. d. Wiss. Göttingen. Math.-nat. Kl. 1911. 1.

OLdHAM, R. D., The Structure of the Himalayas and of the Gangetic Plain as elucidated by geodetic observations in India. Mem. of the Geol. Survey of India. XLII. Pt. II. 1917.

SPENCER, J. W., Relationship between terrestrial gravity and observed earth movements of eastern America. Am. Journ. of Sc. 4. Ser. Vol. XXXV. p. 561.1913.

\title{
Meeresgebiete.
}

HECKER, O., Bestimmungen der Schwerkraft auf dem Schwarzen Meere and an dessen Küste, sowie Ausgleichung der Schwerkraftmessungen auf dem Atlantischen, Indischen und Großen Ozean. Zentralbureau f. internat. Erd. messung. N. F. d. Veröffentl. Nr. 20. Berlin 1910.

WolfF, Hrrmans, Die Sehwerkraft auf dem Meere und die Hypothese von Pratt. Inaug.-Diss. Berlin 1913.

Wolff, HgRmant, Die Schwerkraft auf dem Mittelländischen Meere und die Hypothese von Pratt. Gerlands Beiträge zur Geophysik. Bd. XIV, 3. Leipzig 1916.

\section{Die Ursachen des Bergsturzes am Reintalanger (Wettersteingebirge).}

\author{
Von Dr. Kurt Leuchs in München.
}

Der obere Reintalanger, der Talschlu $B$ der Partnach, war am 6. Mai 1920 Schauplatz eines Bergsturzes. Is war kein durch besondere Größe oder durch verheerende Wirkung auffallender, aber verscbiedene dabei hervortretende Umstände lassen es mir angezeigt erscheinen, diesen Bergsturz kurz. zu beschreiben.

Herr stud. jur. HeinRIce Mosar in München war A ugenzeuge und gab mir folgenden Bericht, wofür ich ihm bestens danke. "Gegen 5 Uhr nachmittags sah er, in der Nähe der Angerhütte stehend, wie sich an einem Felspfeiler östlich des Gatterlbaches eine Felsplatte ablöste und langsam, obne daß Geräusche hörbar waren, gegen den Anger zu neigte. Alsbald zerbrach dis Platte, indem sich zuerst ihr oberer klotziger Teil abtrennte; die ganze Masse stürzte, in sich zusammen. brechend, hinab an den Fuß der Felswände, deren untere, weniger steile Partien glattfegend. Einzelne Blöcke wurden, auf Wandvorsprünge aufschlagend and dort abprallend, weit hinausgeschleudert nach den Seiten und nach vorwärts bis auf die Nordhänge des Angers. Die Hauptmasse stürzte in die Rinne des Gatterlbaches, schob und wälzte sich von dort, vermengt mit dem Neuschnee, weiter als 